PHYSICAL REVIEW D 74, 115015 (2006)

\title{
Light Higgs production in hyperon decay
}

\author{
Xiao-Gang $\mathrm{He}^{*}$ \\ Department of Physics and Center for Theoretical Sciences, National Taiwan University, Taipei, Taiwan \\ Jusak Tandean \\ Department of Mathematics/Physics/Computer Science, University of La Verne, La Verne, California 91750, USA \\ G. Valencia \\ Department of Physics and Astronomy, Iowa State University, Ames, Iowa 50011, USA
}

(Received 30 October 2006; published 22 December 2006)

\begin{abstract}
A recent HyperCP observation of three events in the decay $\Sigma^{+} \rightarrow p \mu^{+} \mu^{-}$is suggestive of a new particle with mass $214.3 \mathrm{MeV}$. In order to confront models that contain a light Higgs boson with this observation, it is necessary to know the Higgs production rate in hyperon decay. The contribution to this rate from penguinlike two-quark operators has been considered before and found to be too large. We point out that there are additional four-quark contributions to this rate that could be comparable in size to the two-quark contributions, and that could bring the total rate to the observed level in some models. To this effect we implement the low-energy theorems that dictate the couplings of light Higgs bosons to hyperons at leading order in chiral perturbation theory. We consider the cases of scalar and pseudoscalar Higgs bosons in the standard model and in its two-Higgs-doublet extensions to illustrate the challenges posed by existing experimental constraints and suggest possible avenues for models to satisfy them.
\end{abstract}

DOI: $10.1103 /$ PhysRevD.74.115015

PACS numbers: 14.80.-j, 11.30.Rd, 12.15.Ji, 13.30.Ce

\section{INTRODUCTION}

Three events for the decay mode $\Sigma^{+} \rightarrow p \mu^{+} \mu^{-}$with a dimuon invariant mass of $214.3 \pm 0.5 \mathrm{MeV}$ have been recently observed by the HyperCP Collaboration [1]. It is possible to account for these events within the standard model (SM) when long-distance contributions are properly included [2]. However, the probability of having all three events at the same dimuon mass in the SM is less than $1 \%$. This suggests a new-particle interpretation for the events, for which the branching ratio is $\left(3.1_{-1.9}^{+2.4} \pm 1.5\right) \times 10^{-8}[1]$.

This possibility has been explored to some extent in the literature, where it has been shown that kaon decays place severe constraints on the couplings of the hypothetical new particle [3-5]. In particular, it was found that the flavorchanging coupling of the new state, $X$, to $\bar{d} s$ has to be of a pseudoscalar or axial-vector nature to explain why the state has not been seen in $K \rightarrow \pi \mu^{+} \mu^{-}$. At least one model containing a particle with these properties has appeared in the literature [6].

All these previous analyses of $X$ considered only the effects of two-quark operators for $\bar{d} s X$. However, it is well known in the case of light Higgs production in kaon decay that there are also four-quark operators that can contribute at the same level as the two-quark ones [7-10]. These fourquark contributions are most conveniently described in chiral perturbation theory $(\chi \mathrm{PT})$ which implements lowenergy theorems governing the couplings of light (pseu-

\footnotetext{
*Electronic address: hexg@phys.ntu.edu.tw

${ }^{\dagger}$ Electronic address: jtandean@ulv.edu

\#Electronic address: valencia@iastate.edu
}

do)scalars to hadrons. In this paper we generalize existing studies appropriate for kaon decay to the case of hyperon decay. This allows us to discuss the production of light (pseudo)scalars in hyperon decay consistently, including the effects of both the two- and four-quark operators with the aid of $\chi \mathrm{PT}$. We consider the cases of scalar and pseudoscalar Higgs bosons in the SM and in the twoHiggs-doublet model (2HDM), expressing our results in a form that can be easily applied to more complicated Higgs models.

This paper is organized as follows. We begin by collecting in Sec. II the existing constraints on light Higgs bosons from kaon, $B$-meson, and hyperon decays if we interpret the HyperCP events as being mediated by a light Higgs boson. In Secs. III and IV we compute the production rates in both kaon and hyperon decays for a light scalar and pseudoscalar Higgs boson, respectively. Finally, in Sec. V we summarize our results and state our conclusions.

\section{SUMMARY OF EXISTING CONSTRAINTS}

In Ref. [3] we parametrized the possible couplings of the new particle, $X$, to $\bar{d} s$ and $\bar{\mu} \mu$ assuming that it had definite parity. Whereas this is a reasonable assumption for the diagonal couplings of $X$ to fermions, it is not for its flavor-changing neutral couplings (FCNCs). Two-quark FCNCs are predominantly induced by Higgs-penguin diagrams, which result in left- and right-handed couplings, implying that the scalar and pseudoscalar ones are present simultaneously. For this reason, we revisit the existing constraints for $X$ being a scalar particle, $\mathcal{H}$, or a pseudoscalar particle, $\mathcal{A}$, assuming them to have two-fermion 
FCNCs described by

$$
\mathcal{L}_{\mathcal{H} s d}=\frac{g_{\mathcal{H}}}{v}\left[m_{s} \bar{d}\left(1+\gamma_{5}\right) s+m_{d} \bar{d}\left(1-\gamma_{5}\right) s\right] \mathcal{H}+\text { H.c. }
$$

$\mathcal{L}_{\mathcal{A} s d}=\frac{i g_{\mathcal{A}}}{v}\left[m_{s} \bar{d}\left(1+\gamma_{5}\right) s-m_{d} \bar{d}\left(1-\gamma_{5}\right) s\right] \mathcal{A}+$ H.c.,

where the $g$ 's are coupling constants, $m_{q}$ is a quark mass, and $v=2^{-1 / 4} G_{F}^{-1 / 2}=246 \mathrm{GeV}$. In addition, the diagonal couplings to charged leptons are assumed to have definite parity and be proportional to the lepton mass,

$$
\mathcal{L}_{\mathcal{H} \ell}=\frac{g_{\ell} m_{\ell}}{v} \bar{\ell} \ell \mathcal{H}, \quad \mathcal{L}_{\mathcal{A} \ell}=\frac{i g_{\ell} m_{\ell}}{v} \bar{\ell} \gamma_{5} \ell \mathcal{A} .
$$

For a (pseudo)scalar of mass $214.3 \mathrm{MeV}$, it is then natural to assume that the decay $X \rightarrow \mu^{+} \mu^{-}$will dominate over the other kinematically allowed modes: $X \rightarrow$ $e^{+} e^{-}, \nu \bar{\nu}, \gamma \gamma$. We will restrict ourselves to this case, assuming that $\mathcal{B}\left(X \rightarrow \mu^{+} \mu^{-}\right) \sim 1$. This is true, for example, for a light SM Higgs boson where $g_{\ell}=1$, or for light pseudoscalars in the 2HDM types I and II, where $g_{\ell}=\cot \beta$ and $-\tan \beta$, respectively. In all these cases, $X \rightarrow e^{+} e^{-}$are suppressed at least by $\left(m_{e} / m_{\mu}\right)^{2} \sim 10^{-5}$.

To be consistent with the HyperCP observation, $X$ must be short lived and decay inside the detector. This is compatible with the estimate for the total width $\Gamma_{\mathcal{A}} \sim$ $10^{-7} \mathrm{MeV}$ [5] of a pseudoscalar particle, $\mathcal{A}$. It was shown in Ref. [3] that the muon anomalous magnetic moment imposes the constraint

$$
\left|g_{\ell}\right| \lesssim 1.2 .
$$

A coupling satisfying this constraint implies a width

$$
\Gamma_{\mathcal{A}} \lesssim 3.7 \times 10^{-7} \mathrm{MeV},
$$

consistent with the observation. In contrast, the corresponding constraint for a scalar particle is $\left|g_{\ell}\right| \lesssim 0.98$, leading to a longer lifetime,

$$
\Gamma_{\mathcal{H}} \lesssim 6.9 \times 10^{-9} \mathrm{MeV} .
$$

The estimated lifetime for the HyperCP particle is therefore consistent with that of a pseudoscalar or scalar that decays predominantly into muons.

In addition to the two-quark contributions to the amplitudes for $K \rightarrow \pi \mathcal{H}(\mathcal{A})$ and $\Sigma^{+} \rightarrow p \mathcal{H}(\mathcal{A})$ induced by the interactions in Eq. (1), we will also include contributions arising from the usual SM four-quark $|\Delta S|=1$ operators, along with flavor-conserving couplings of $\mathcal{H}(\mathcal{A})$. We will adopt the chiral-Lagrangian approach to evaluate the hadron-level interactions.

Later on we will discuss specific models and consider the bounds appropriate for them, including all the relevant two- and four-quark contributions. It is useful to start with one example to illustrate the ingredients needed to con- struct a model that can satisfy all the existing constraints. For this purpose, we consider a pseudoscalar $\mathcal{A}$ with twoquark couplings as in Eq. (1b) supplemented with simple parametrizations for the four-quark amplitudes for both kaon and hyperon decays. For $B$-meson decay, we assume that the two-quark contribution completely dominates.

\section{A. $K \rightarrow \pi \mathcal{A}$}

Introducing the dimensionless quantity $M_{4 K}$ for the fourquark contribution, we express the amplitude for $K^{ \pm} \rightarrow$ $\pi^{ \pm} \mathcal{A}$ and its branching ratio, respectively, as

$$
\begin{aligned}
i \mathcal{M}\left(K^{ \pm} \rightarrow \pi^{ \pm} \mathcal{A}\right) & =g_{\mathcal{A}} \frac{m_{K}^{2}-m_{\pi}^{2}}{v}-M_{4 K} \frac{m_{K}^{2}}{v}, \\
\mathcal{B}\left(K^{ \pm} \rightarrow \pi^{ \pm} \mathcal{A}\right) & =4.43 \times 10^{8}\left|g_{\mathcal{A}}-1.08 M_{4 K}\right|^{2} .
\end{aligned}
$$

This mode is constrained by its nonobservation in the BNL E865 [11] or FNAL HyperCP [12] measurements of $K^{ \pm} \rightarrow$ $\pi^{ \pm} \mu^{+} \mu^{-}$. It is also constrained by its nonobservation in the isospin-related mode $K_{S} \rightarrow \pi^{0} \mu^{+} \mu^{-}$by CERN NA48 [13]. Of these three experiments, E865 had the best statistics, collecting 430 events in $K^{+} \rightarrow \pi^{+} \mu^{+} \mu^{-}$. A new particle $\mathcal{A}$ of mass $214.3 \mathrm{MeV}$ would have contributed only in their first dimuon-mass bin, where $0.21 \mathrm{GeV}<$ $m_{\mu \mu}<0.224 \mathrm{GeV}$ and approximately 30 events were observed. To obtain a conservative bound, we assume that all the events in the first bin are statistically Gaussian and can be attributed to the new particle (either a scalar or a pseudoscalar). Further assuming uniform acceptance, we obtain at $95 \%$ C.L.

$$
\mathcal{B}\left(K^{+} \rightarrow \pi^{+} X\right) \lesssim 8.7 \times 10^{-9} .
$$

The NA48 Collaboration collected 6 events for $K_{S} \rightarrow$ $\pi^{0} \mu^{+} \mu^{-}$[13], and none of them have the $214.3 \mathrm{MeV}$ invariant mass required if they originate from the new particle $\mathcal{A}$. Using the $K_{S}$ flux and the acceptance at low $m_{\mu \mu}$ in Ref. [13], we estimate a single event sensitivity of $\left(5.3_{-0.4}^{+0.6}\right) \times 10^{-10}$. With no events observed and Poisson statistics, this translates into the 95\%-C.L. bound

$$
\mathcal{B}\left(K_{S} \rightarrow \pi^{0} X\right) \lesssim 1.8 \times 10^{-9} .
$$

We employ these bounds when we discuss specific models, but for now we use the E865 result in Eq. (7), combined with Eq. (6), to find

$$
\begin{gathered}
\left|g_{\mathcal{A}}-1.08 M_{4 K}\right| \lesssim 4.4 \times 10^{-9} . \\
\text { B. } \boldsymbol{\Sigma}^{+} \rightarrow \boldsymbol{p} \mathcal{A}
\end{gathered}
$$

In this case, we need two new dimensionless quantities $A_{4}$ and $B_{4}$ to parametrize the effect of the four-quark operators, writing the amplitude as

$$
\mathcal{M}\left(\Sigma^{+} \rightarrow p \mathcal{A}\right)=i \bar{p}\left(A_{p \mathcal{A}}-B_{p \mathcal{A}} \gamma_{5}\right) \Sigma^{+},
$$

where 


$$
\begin{aligned}
& A_{p \mathcal{A}}=g_{\mathcal{A}} \frac{m_{\Sigma}-m_{N}}{v}+A_{4} \frac{f_{\pi}}{v}, \\
& B_{p \mathcal{A}}=g_{\mathcal{A}}(D-F) \frac{m_{\Sigma}+m_{N}}{v} \frac{m_{K}^{2}}{m_{K}^{2}-m_{\mathcal{A}}^{2}}+B_{4} \frac{f_{\pi}}{v},
\end{aligned}
$$

the parameters $D$ and $F$ coming from a chiral Lagrangian to be discussed in a later section and $f_{\pi}=92.4 \mathrm{MeV}$ being the pion-decay constant. The resulting branching ratio is

$$
\begin{aligned}
\mathcal{B}\left(\Sigma^{+} \rightarrow p \mathcal{A}\right)= & 1.91 \times 10^{6}\left|g_{\mathcal{A}}+0.36 A_{4}\right|^{2} \\
& +4.84 \times 10^{4}\left|g_{\mathcal{A}}+0.14 B_{4}\right|^{2}
\end{aligned}
$$

with the choice $D-F=0.25$. Combining the statistical and systematic errors of the HyperCP measurement [1] in quadrature, we require

$$
\mathcal{B}\left(\Sigma^{+} \rightarrow p \mathcal{A}\right)=\left(3.1_{-2.4}^{+2.8}\right) \times 10^{-8},
$$

and therefore

$$
\left|g_{\mathcal{A}}+0.36 A_{4}\right|=(1.3 \pm 0.6) \times 10^{-7},
$$

where we have used the larger of the errors in Eq. (12) and ignored the contribution from the $P$-wave term in Eq. (11), assuming that $B_{4} \lesssim A_{4}$. This assumption is satisfied by all the models we discuss, but when checking a specific model, we do so without neglecting $B_{4}$.

A comparison of Eqs. (9) and (13) shows why it is not possible to have a (pseudo)scalar with penguinlike flavorchanging neutral couplings, as in Eq. (1), as an explanation for the HyperCP result given the constraints from kaon decay. It also shows how this is no longer true if there are four-quark contributions to the amplitudes that are comparable to the penguin amplitudes. In particular, if we assume that in a given model $g_{\mathcal{A}}, M_{4 K}$, and $A_{4}$ have comparable magnitudes, we see that in order to satisfy both Eqs. (9) and (13) we need a cancellation between the two- and fourquark contributions to the kaon amplitude that reduces them by a factor of about 20 . As we will show in later sections, this is possible in many models. For this cancellation to work, however, $g_{\mathcal{A}}$ and $M_{4 K}$ must also have similar phases. As we will see, this is a requirement that is much harder to satisfy. In the simple models we consider in this paper, the phase of $g_{\mathcal{A}}$ is much larger than the phase of $M_{4 K}$ so that the cancellation does not happen for the imaginary part.

\section{C. $b \rightarrow s X$}

Finally, we consider the constraints on the new particle from its nonobservation in $B$-meson decay. In this case, the four-quark contributions are negligible, and we can neglect $m_{s}$ compared to $m_{b}$. The Lagrangian for $b \rightarrow s X$ can then be expressed as

$$
\mathcal{L}_{X b s}=\frac{g^{\prime} m_{b}}{v} \bar{s}\left(1+\gamma_{5}\right) b X+\text { H.c., }
$$

where $g^{\prime}=g_{\mathcal{H}}^{\prime}\left(i g_{\mathcal{A}}^{\prime}\right)$ for $X=\mathcal{H}(\mathcal{A})$. This leads to the partial decay rate

$$
\Gamma(b \rightarrow s X) \simeq\left|g^{\prime}\right|^{2} \frac{m_{b}^{3}}{8 \pi v^{2}} .
$$

Using for illustration $m_{b}=4.3 \mathrm{GeV}$ and the $B^{+}$lifetime [14] results in

$$
\mathcal{B}(b \rightarrow s X)=1.3 \times 10^{8}\left|g^{\prime}\right|^{2} .
$$

One could obtain a similar number for $b \rightarrow d X$.

The latest experimental average $\mathcal{B}\left(b \rightarrow s \mu^{+} \mu^{-}\right)=$ $\left(4.27_{-1.22}^{+1.23}\right) \times 10^{-6}$ [14] covers the full kinematic range for $m_{\mu \mu}$. To constrain $g^{\prime}$, it is better to limit the comparison to the measured rate at the lowest measured $m_{\mu \mu}$ invariantmass bin. BABAR quotes in Table 2 of Ref. [15]

$$
\begin{aligned}
\mathcal{B}(b & \left.\rightarrow s \ell^{+} \ell^{-}\right)_{m_{\ell^{+} \ell^{-}} \in[0.2 \mathrm{GeV}, 1.0 \mathrm{GeV}]} \\
& =\left(0.08 \pm 0.36_{-0.04}^{+0.07}\right) \times 10^{-6} .
\end{aligned}
$$

This is an average for electrons and muons, but no noticeable difference between them was found. Belle quotes on Table 4 of Ref. [16] the corresponding number

$$
\begin{aligned}
\mathcal{B}(b & \left.\rightarrow s \ell^{+} \ell^{-}\right)_{m_{\ell^{+} \ell^{-}} \in[0.2 \mathrm{GeV}, 1.0 \mathrm{GeV}]} \\
& =\left(11.3 \pm 4.8_{-2.7}^{+4.6}\right) \times 10^{-7} .
\end{aligned}
$$

To be conservative, we constrain the Higgs coupling by requiring that the induced rate be below the 95\%-C.L. upper range of the measured $b \rightarrow s \ell^{+} \ell^{-}$rate in the lowest measured $m_{\mu \mu}$ bin. Thus, combining errors in quadrature for the more restrictive $B A B A R$ result gives

$$
\mathcal{B}\left(b \rightarrow s \ell^{+} \ell^{-}\right)_{m_{\ell^{+} \ell^{-}}<1 \mathrm{GeV}} \lesssim 8.0 \times 10^{-7}
$$

and, correspondingly,

$$
\left|g^{\prime}\right| \lesssim 7.8 \times 10^{-8} .
$$

The exclusive $B \rightarrow\left(K, K^{\star}\right) \mu^{+} \mu^{-}$modes have been measured, but the resulting constraints are not better than Eq. (20). This constraint, Eq. (20), is difficult to satisfy in models where $g_{\mathcal{A}}$ and $g^{\prime}$ are related by top-quark Cabibbo-Kobayashi-Maskawa (CKM) angles, as happens in the simple models we consider here.

\section{SCALAR HIGGS BOSON}

In this section we discuss in detail the case of a light Higgs boson in the standard model and in the two-Higgsdoublet model. We will use known low-energy theorems to implement the four-quark contributions to kaon and hyperon amplitudes. 


\section{A. Two-quark $|\Delta S|=1$ interactions}

The effective Lagrangian for the $s d \mathcal{H}$ coupling, where $\mathcal{H}$ is either the standard-model Higgs boson $H^{0}$ or the lightest scalar Higgs boson $h^{0}$ in the 2HDM, has been much discussed in the literature [7,8,17-19] and can be written as $\mathcal{L}_{\mathcal{H} s d}$ in Eq. (1a), where

$$
g_{\mathcal{H}}=\frac{G_{F}}{4 \sqrt{2} \pi^{2}} \sum_{q=u, c, t} m_{q}^{2} V_{q d}^{*} V_{q s} F(q),
$$

with $V_{k l}$ being the elements of the CKM matrix and $F(q)$ depending on the model. In the SM, for a Higgs mass much smaller than the $W$ mass,

$$
F(q)=3 / 4,
$$

whereas in the 2HDM the expression for $F(q)$ is much lengthier $[18,19]$.

Using CKM and mass parameters from Ref. [20], we find in the SM

$$
g_{\mathcal{H}}=(-1.3-0.6 i) \times 10^{-6},
$$

to be compared with Eqs. (9) and (13) above. Employing the expression for $F(q)$ derived in Ref. [19], we obtain a similar number in the 2HDM type II, for instance,

$$
g_{\mathcal{H}}=(5.0+1.9 i) \times 10^{-7}
$$

for the parameters

$$
\begin{gathered}
\tan \beta \simeq 2.57, \quad \sin (\beta-\alpha) \simeq 0.149, \\
m_{H^{+}}=250 \mathrm{GeV},
\end{gathered}
$$

where $\tan \beta$ is the ratio of vacuum expectation values of the two Higgs doublets, $\alpha$ the mixing angle in the neutralHiggs-boson mass matrix, and $m_{H^{+}}$the mass of the charged Higgs bosons. ${ }^{1}$ We note that the $\alpha$ and $\beta$ values above satisfy the constraint $\sin ^{2}(\beta-\alpha)<0.06$ from CERN LEP [21]. We see right away that $g_{\mathcal{H}}$ can be in the right ball park to explain the HyperCP observation, Eq. (13), but conflicts with the kaon bound, Eq. (9).

To evaluate the hadronic amplitudes from this two-quark contribution, we employ chiral perturbation theory. Using the operator matching of Ref. [3], we write the lowestorder chiral realization of $\mathcal{L}_{\mathcal{H} s d}$ as

$$
\begin{aligned}
\mathcal{L}_{\mathcal{H}}= & b_{D}\left\langle\bar{B}\left\{h_{\mathcal{H}}, B\right\}\right\rangle+b_{F}\left\langle\bar{B}\left[h_{\mathcal{H}}, B\right]\right\rangle+b_{0}\left\langle h_{\mathcal{H}}\right\rangle\langle\bar{B} B\rangle \\
& +\frac{1}{2} f^{2} B_{0}\left\langle h_{\mathcal{H}}\right\rangle+\text { H.c. }
\end{aligned}
$$

where $\langle\cdots\rangle \equiv \operatorname{Tr}(\cdots)$ in flavor-SU(3) space, $f=f_{\pi}=$ 92.4 $\mathrm{MeV}$, and

$$
h_{\mathcal{H}}=-2 g_{\mathcal{H}}\left(\xi^{\dagger} h M \xi^{\dagger}+\xi M h \xi\right) \frac{\mathcal{H}}{v},
$$

with $h$ being a $3 \times 3$ matrix having elements $h_{k l}=\delta_{k 2} \delta_{3 l}$

\footnotetext{
${ }^{1}$ We have also set $\kappa=m_{H^{+}}^{2} / m_{W}^{2}$ in $F(q)$, where $\kappa$ is defined in Ref. [19].
}

which selects out $s \rightarrow d$ transitions, $\quad M=$ $\operatorname{diag}\left(\hat{m}, \hat{m}, m_{s}\right)=\operatorname{diag}\left(m_{\pi}^{2}, m_{\pi}^{2}, 2 m_{K}^{2}-m_{\pi}^{2}\right) /\left(2 B_{0}\right) \quad$ the quark-mass matrix in the isospin-symmetric limit $m_{u}=$ $m_{d}=\hat{m}$, and the baryon and meson fields represented by the usual $3 \times 3$ matrices $B$ and $\Sigma=\xi \xi=e^{i \varphi / f}$, respectively.

To derive amplitudes, we also need the chiral Lagrangian for the strong interactions of the hadrons $[22,23]$. At leading order in the derivative and $m_{s}$ expansions, it can be written as

$$
\begin{aligned}
\mathcal{L}_{\mathrm{s}}= & \left\langle\bar{B} i \gamma^{\mu}\left(\partial_{\mu} B+\left[\mathcal{V}_{\mu}, B\right]\right)\right\rangle-m_{0}\langle\bar{B} B\rangle \\
& +D\left\langle\bar{B} \gamma^{\mu} \gamma_{5}\left\{\mathcal{A}_{\mu}, B\right\}\right\rangle+F\left\langle\bar{B} \gamma^{\mu} \gamma_{5}\left[\mathcal{A}_{\mu}, B\right]\right\rangle \\
& +b_{D}\left\langle\bar{B}\left\{M_{+}, B\right\}\right\rangle+b_{F}\left\langle\bar{B}\left[M_{+}, B\right]\right\rangle+b_{0}\left\langle M_{+}\right\rangle\langle\bar{B} B\rangle \\
& +\frac{1}{4} f^{2}\left\langle\partial^{\mu} \Sigma^{\dagger} \partial_{\mu} \Sigma\right\rangle+\frac{1}{2} f^{2} B_{0}\left\langle M_{+}\right\rangle,
\end{aligned}
$$

where $\mathcal{V}^{\mu}=\frac{1}{2}\left(\xi \partial^{\mu} \xi^{\dagger}+\xi^{\dagger} \partial^{\mu} \xi\right), m_{0}$ is the baryon mass in the chiral limit, $\mathcal{A}^{\mu}=\frac{i}{2}\left(\xi \partial^{\mu} \xi^{\dagger}-\xi^{\dagger} \partial^{\mu} \xi\right)$, and $M_{+}=$ $\xi^{\dagger} M \xi^{\dagger}+\xi M^{\dagger} \xi$, with further details being given in Ref. [3].

From $\mathcal{L}_{\mathcal{H}}$ and $\mathcal{L}_{\mathrm{s}}$, we derive the leading-order diagrams shown in Fig. 1 for $\Sigma^{+} \rightarrow p \mathcal{H}$, yielding the amplitude

$$
\begin{aligned}
\mathcal{M}_{2 q}\left(\Sigma^{+} \rightarrow p \mathcal{H}\right)= & g_{\mathcal{H}} \frac{m_{\Sigma}-m_{N}}{v} \frac{m_{K}^{2}}{m_{K}^{2}-m_{\pi}^{2}} \bar{p} \Sigma^{+} \\
& -g_{\mathcal{H}}(D-F) \frac{m_{\Sigma}+m_{N}}{v} \frac{m_{K}^{2}-m_{\pi}^{2}}{m_{K}^{2}-m_{\mathcal{H}}^{2}} \\
& \times \bar{p} \gamma_{5} \Sigma^{+},
\end{aligned}
$$

where the two terms correspond to the two diagrams, respectively, $m_{\Sigma, N}$ are isospin-symmetric masses, and we have used the relations $m_{\Sigma}-m_{N}=2\left(b_{D}-b_{F}\right)\left(m_{s}-\hat{m}\right)$, $m_{K}^{2}=B_{0}\left(\hat{m}+m_{s}\right)$, and $m_{\pi}^{2}=2 B_{0} \hat{m}$ derived from $\mathcal{L}_{\mathrm{s}}$. Numerically, we will allow $D$ and $F$ to have the ranges $0.6 \leq D \leq 0.8$ and $0.4 \leq F \leq 0.5$ [23], leading to

$$
0.1 \leq D-F \leq 0.4 \text {, }
$$

which is their combination occurring in our amplitudes.

It follows that the contribution of $\mathcal{L}_{\mathcal{H} s d}$ to the branching ratio of $\Sigma^{+} \rightarrow p \mathcal{H}$ for $m_{\mathcal{H}}=214.3 \mathrm{MeV}$ and the middle value $D-F=0.25$ is in the SM

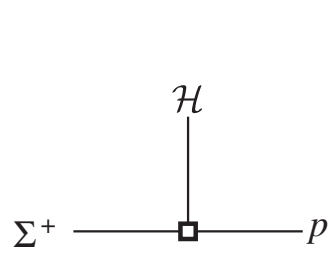

(a)

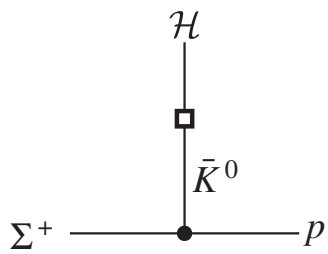

(b)
FIG. 1. Diagrams contributing to $\Sigma^{+} \rightarrow p \mathcal{H}$ arising from $\mathcal{L}_{\mathcal{H} s d}$ at leading order in $\chi \mathrm{PT}$. The square vertices come from $\mathcal{L}_{\mathcal{H}}$ in Eq. (26), and the solid vertex from $\mathcal{L}_{\mathrm{s}}$ in Eq. (28). 


$$
\mathcal{B}_{2 q}\left(\Sigma^{+} \rightarrow p \mathcal{H}\right)=(40+1) \times 10^{-7},
$$

where we have ignored the imaginary ( $C P$-violating) part of the amplitude, and the two numbers correspond to the contributions from the scalar and pseudoscalar flavorchanging couplings, respectively. Evidently, the scalar contribution is much larger than what HyperCP saw, but the pseudoscalar contribution is within the range. This, however, is only part of the story, as there are in addition four-quark contributions to be discussed in the next subsection.

Also from $\mathcal{L}_{\mathcal{H}}$, we derive the leading-order diagram for $K \rightarrow \pi \mathcal{H}$, which is that in Fig. 1(a) with $\Sigma^{+}(p)$ replaced by $K(\pi)$ and arises from the scalar coupling in $\mathcal{L}_{\mathcal{H} s d}$. The resulting amplitude is

$$
\begin{aligned}
\mathcal{M}_{2 q}\left(K^{+} \rightarrow \pi^{+} \mathcal{H}\right) & =-\sqrt{2} \mathcal{M}_{2 q}\left(K^{0} \rightarrow \pi^{0} \mathcal{H}\right) \\
& =\frac{-g_{\mathcal{H}} m_{K}^{2}}{v}
\end{aligned}
$$

and $\quad$ so $\mathcal{M}_{2 q}\left(K_{L} \rightarrow \pi^{0} \mathcal{H}\right)=-\operatorname{Re} \mathcal{M}_{2 q}\left(K^{+} \rightarrow \pi^{+} \mathcal{H}\right)$. Dropping again the imaginary parts of the amplitudes, we obtain in the SM the branching ratios

$$
\begin{aligned}
\mathcal{B}_{2 q}\left(K^{+} \rightarrow \pi^{+} \mathcal{H}\right) & =9.3 \times 10^{-4}, \\
\mathcal{B}_{2 q}\left(K_{L} \rightarrow \pi^{0} \mathcal{H}\right) & =3.9 \times 10^{-3} .
\end{aligned}
$$

These numbers would easily be incompatible with that in Eq. (7) and the 95\%-C.L. bound ${ }^{2}$

$$
\mathcal{B}\left(K_{L} \rightarrow \pi^{0} \mu^{+} \mu^{-}\right)<4.9 \times 10^{-10},
$$

but, as in the $\Sigma^{+}$case, there are four-quark contributions that have to be considered as well.

The situation is similar in the 2HDM. Adopting the real part of the coupling in Eq. (24), for example, we find

$$
\begin{aligned}
\mathcal{B}_{2 q}\left(\Sigma^{+} \rightarrow p \mathcal{H}\right) & =(56+1) \times 10^{-8}, \\
\mathcal{B}_{2 q}\left(K^{+} \rightarrow \pi^{+} \mathcal{H}\right) & =1.3 \times 10^{-4}, \\
\mathcal{B}_{2 q}\left(K_{L} \rightarrow \pi^{0} \mathcal{H}\right) & =5.4 \times 10^{-4} .
\end{aligned}
$$

\section{B. Four-quark $|\Delta S|=1$ interactions}

The hadronic interactions of a light Higgs boson due to four-quark $|\Delta S|=1$ operators are best accounted for in the chiral-Lagrangian approach. The dominant contribution is generated by the $|\Delta I|=\frac{1}{2}$ component of the effective Hamiltonian transforming as $\left(8_{\mathrm{L}}, 1_{\mathrm{R}}\right)$. The corresponding Lagrangian at leading order is given by $[23,25]$

\footnotetext{
${ }^{2}$ We have inferred this number from Ref. [24] which reported $\mathcal{B}\left(K_{L} \rightarrow \pi^{0} \mu^{+} \mu^{-}\right)<3.8 \times 10^{-10}$ at $90 \%$ C.L.
}

$$
\begin{aligned}
\mathcal{L}_{\mathrm{w}}= & h_{D}\left\langle\bar{B}\left\{\xi^{\dagger} h \xi, B\right\}\right\rangle+h_{F}\left\langle\bar{B}\left[\xi^{\dagger} h \xi, B\right]\right\rangle \\
& +\gamma_{8} f^{2}\left\langle h \partial_{\mu} \Sigma \partial^{\mu} \Sigma^{\dagger}\right\rangle+2 \tilde{\gamma}_{8} f^{2} B_{0}\left\langle h \xi M_{+} \xi^{\dagger}\right\rangle \\
& + \text { H.c., }
\end{aligned}
$$

where $h_{D, F}$ can be extracted from hyperon nonleptonic decays, $\gamma_{8}=-7.8 \times 10^{-8}$ from $K \rightarrow \pi \pi$, the sign following from various predictions $[7-9,26]$, and $\tilde{\gamma}_{8}$ is unknown as it does not contribute to any process with only kaons and pions.

The four-quark $|\Delta S|=1$ interactions of a light Higgs boson $\mathcal{H}$ arise from its tree-level couplings to quarks and $W^{ \pm}$bosons, as well as from its coupling to gluons induced by a triangle diagram with heavy quarks in the loop. To obtain the relevant chiral Lagrangians, one starts with $\mathcal{L}_{\mathrm{s}, \mathrm{w}}$ above and follows the prescription given in Refs. [7-9,19]. The results are

$$
\begin{aligned}
\mathcal{L}_{\mathrm{s}}^{\mathcal{H}}= & \left(\frac{1}{4} c_{1} f^{2}\left\langle\partial^{\mu} \Sigma^{\dagger} \partial_{\mu} \Sigma\right\rangle+\frac{1}{2} c_{2} f^{2} B_{0}\left\langle M_{+}\right\rangle\right. \\
& \left.+\frac{1}{2} f^{2} B_{0}\left\langle\hat{M}_{+}-M_{+}\right\rangle\right) \frac{\mathcal{H}}{v}-k_{1} m_{0}\langle\bar{B} B\rangle \frac{\mathcal{H}}{v} \\
& +k_{2}\left(b_{D}\left\langle\bar{B}\left\{\hat{M}_{+}, B\right\}\right\rangle+b_{F}\left\langle\bar{B}\left[\hat{M}_{+}, B\right]\right\rangle\right. \\
& \left.+b_{0}\left\langle\hat{M}_{+}\right\rangle\langle\bar{B} B\rangle\right) \frac{\mathcal{H}}{v},
\end{aligned}
$$

$$
\begin{aligned}
\mathcal{L}_{\mathrm{w}}^{\mathcal{H}}= & {\left[\gamma_{8} c_{3} f^{2}\left\langle h \partial_{\mu} \Sigma \partial^{\mu} \Sigma^{\dagger}\right\rangle+2 \tilde{\gamma}_{8} c_{4} f^{2} B_{0}\left\langle h \xi M_{+} \xi^{\dagger}\right\rangle\right.} \\
& \left.+2 \tilde{\gamma}_{8} f^{2} B_{0}\left\langle h \xi\left(\hat{M}_{+}-M_{+}\right) \xi^{\dagger}\right\rangle\right] \frac{\mathcal{H}}{v} \\
& +k_{3}\left(h_{D}\left\langle\bar{B}\left\{\xi^{\dagger} h \xi, B\right\}\right\rangle+h_{F}\left\langle\bar{B}\left[\xi^{\dagger} h \xi, B\right]\right\rangle\right) \frac{\mathcal{H}}{v} \\
& + \text { H.c., }
\end{aligned}
$$

where

$c_{1}=2 k_{G}, \quad c_{2}=3 k_{G}+1, \quad c_{3}=4 k_{G}-2 k_{W}$,

$c_{4}=5 k_{G}-2 k_{W}+1, \quad k_{1}=k_{G}, \quad k_{2}=1$,

$k_{3}=3 k_{G}-2 k_{W}, \quad \hat{M}_{+}=\xi^{\dagger} \hat{M} \xi^{\dagger}+\xi \hat{M}^{\dagger} \xi$,

with

$$
k_{G}=\frac{2\left(2 k_{u}+k_{d}\right)}{27}, \quad \hat{M}=\operatorname{diag}\left(k_{u} \hat{m}, k_{d} \hat{m}, k_{d} m_{s}\right)
$$

the expression for $k_{G}$ corresponding to 3 heavy and 3 light quarks. The parameters $k_{u, d}, k_{W}$, and $k_{G}$ come from the couplings of $\mathcal{H}$ to light quarks, $W^{ \pm}$, and the gluons, respectively, and depend on the model of the Higgs sector. Thus

$$
k_{u}=k_{d}=k_{W}=1 \quad \text { in the } \mathrm{SM},
$$




$$
k_{u}=k_{d}=\frac{\cos \alpha}{\sin \beta}, \quad k_{W}=\sin (\beta-\alpha) \quad \text { in the 2HDM I, }
$$

$$
\begin{aligned}
& k_{u}=\frac{\cos \alpha}{\sin \beta}, \quad k_{d}=-\frac{\sin \alpha}{\cos \beta}, \\
& k_{W}=\sin (\beta-\alpha) \quad \text { in the 2HDM II. }
\end{aligned}
$$

The parameters $c_{1,2,3,4}$ for the meson terms have already been obtained in the literature [7-9,19,27], whereas the new ones $k_{1,2,3}$ follow from how the baryon parameters depend on masses: $m_{0} \sim \Lambda, b_{D, F, 0} \sim 1, \chi_{+} \sim \Lambda m_{q}$, and $h_{D, F} \sim \Lambda^{3} / m_{W}^{2}$, where $\Lambda$ is a QCD mass scale. Note that we work in that basis in which the mass terms in the Lagrangians are not diagonal and must therefore include the corresponding tadpole diagrams in our calculation.

For $\Sigma^{+} \rightarrow p \mathcal{H}$, we derive from $\mathcal{L}_{\mathrm{s}, \mathrm{w}}^{(\mathcal{H})}$ the diagrams shown in Fig. 2, finding

$$
\begin{aligned}
\mathcal{M}_{4 q}\left(\Sigma^{+} \rightarrow p \mathcal{H}\right)= & \left(k_{d}-3 k_{G}+2 k_{W}\right) \frac{h_{D}-h_{F}}{v} \bar{p} \Sigma^{+} \\
& +4\left(k_{G}-k_{W}\right)(D-F) \tilde{\gamma}_{8} \\
& \times \frac{m_{\Sigma}+m_{N}}{v} \frac{m_{K}^{2}-m_{\pi}^{2}}{m_{K}^{2}-m_{\mathcal{H}}^{2}} \bar{p} \gamma_{5} \Sigma^{+},
\end{aligned}
$$

where the first term comes from the upper three diagrams, which are at leading order, and the second term results from the lower two diagrams, which are at next-to-leading order. Now, the combination $h_{D}-h_{F}$ also occurs in the amplitude for $\Sigma^{+} \rightarrow p \pi^{0}$, which we write as

$$
\mathcal{M}\left(\Sigma^{+} \rightarrow p \pi^{0}\right)=i \bar{p}\left(A_{p \pi^{0}}-B_{p \pi^{0}} \gamma_{5}\right) \Sigma^{+},
$$

where from $\mathcal{L}_{\mathrm{s}, \mathrm{w}}$

$$
\begin{aligned}
A_{p \pi^{0}} & =\frac{-h_{D}+h_{F}}{2 f}, \\
B_{p \pi^{0}} & =(D-F) \frac{h_{D}-h_{F}}{2 f} \frac{m_{\Sigma}+m_{N}}{m_{\Sigma}-m_{N}} .
\end{aligned}
$$

Since from experiment [28]

$$
A_{p \pi^{0}}=-3.25 \times 10^{-7}, \quad B_{p \pi^{0}}=26.67 \times 10^{-7},
$$

up to an overall sign, in our numerical evaluation of the four-quark contributions to $\Sigma^{+} \rightarrow p \mathcal{H}$ we will explore different $h_{D}-h_{F}$ values accordingly.

We can also derive from $\mathcal{L}_{\mathrm{s}, \mathrm{w}}^{(\mathcal{H})}$ the corresponding leading-order diagrams for $K \rightarrow \pi \mathcal{H}$, which are the upper three in Fig. 2 with $\Sigma^{+}(p)$ replaced by $K(\pi)$ and yield

$$
\begin{aligned}
\mathcal{M}_{4 q}\left(K^{+} \rightarrow \pi^{+} \mathcal{H}\right)= & \frac{\gamma_{8}}{v}\left[2\left(k_{W}-k_{G}\right)\left(m_{K}^{2}+m_{\pi}^{2}-m_{\mathcal{H}}^{2}\right)\right. \\
& \left.+\left(k_{d}-k_{u}\right) m_{\pi}^{2}\right] \\
& +\frac{\tilde{\gamma}_{8}}{v} 4\left(k_{G}-k_{W}\right) m_{K}^{2} \\
\mathcal{M}_{4 q}\left(K^{0} \rightarrow \pi^{0} \mathcal{H}\right)= & \frac{\gamma_{8}}{\sqrt{2} v}\left[2\left(k_{G}-k_{W}\right)\left(m_{K}^{2}+m_{\pi}^{2}-m_{\mathcal{H}}^{2}\right)\right. \\
& \left.+\left(k_{u}-k_{d}\right) \frac{m_{\pi}^{2} m_{K}^{2}}{m_{K}^{2}-m_{\pi}^{2}}\right] \\
& +\frac{\tilde{\gamma}_{8}}{\sqrt{2} v}\left[4\left(k_{W}-k_{G}\right) m_{K}^{2}\right. \\
& \left.+\left(k_{d}-k_{u}\right) \frac{m_{\pi}^{2} m_{K}^{2}}{m_{K}^{2}-m_{\pi}^{2}}\right]
\end{aligned}
$$

Since $\tilde{\gamma}_{8}$ is unknown, in evaluating its effect on $K^{+} \rightarrow$ $\pi^{+} \mathcal{H}$ we will allow it to vary from -10 to 10 times $\gamma_{8}$. Naively we would expect $\gamma_{8}$ and $\tilde{\gamma}_{8}$ to be of the same order.

\section{Total contributions}

The total amplitude for $K^{+} \rightarrow \pi^{+} \mathcal{H}$ comes from the sum of the contributions in Eqs. (32) and (48). If the $C P$-violating terms in the amplitudes are ignored, it is possible for the two-quark and four-quark contributions to cancel. We show this possibility in Fig. 3, where we plot the resulting branching ratio in the SM as a function of the ratio $r_{8} \equiv \tilde{\gamma}_{8} / \gamma_{8}$ for $m_{\mathcal{H}}=214.3 \mathrm{MeV}$. We find that $\mathcal{B}\left(K^{+} \rightarrow \pi^{+} \mathcal{H}\right)=0$ when $r_{8} \simeq-5.1$ and that, as the figure indicates, for only a very narrow range of $r_{8}$ around this value does the branching ratio ever fall below the upper limit in Eq. (7). In Fig. 3, we also plot the corre-
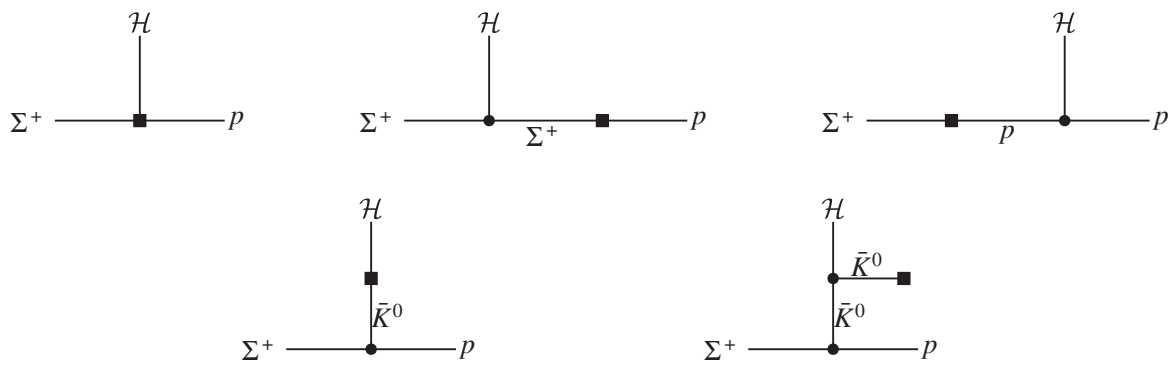

FIG. 2. Diagrams contributing to $\Sigma^{+} \rightarrow p \mathcal{H}$ arising from the four-quark operators. The square vertices come from $\mathcal{L}_{\mathrm{w}}^{(\mathcal{H})}$ in Eqs. (36) and (38), whereas the dots are from $\mathcal{L}_{\mathrm{s}}^{(\mathcal{H})}$ in Eqs. (28) and (37). 
LIGHT HIGGS PRODUCTION IN HYPERON DECAY

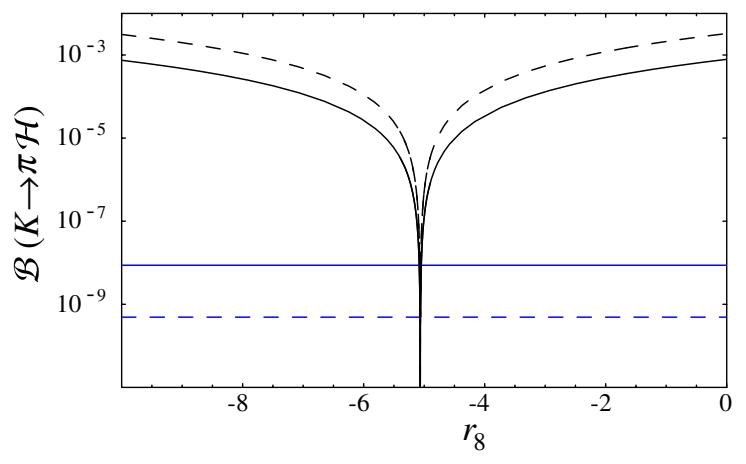

FIG. 3 (color online). Contributions of real parts of total amplitudes for $K^{+} \rightarrow \pi^{+} \mathcal{H}$ (solid curve) and $K_{L} \rightarrow \pi^{0} \mathcal{H}$ (dashed curve) in the SM to their branching ratios as functions of $r_{8}=\tilde{\gamma}_{8} / \gamma_{8}$ for $m_{\mathcal{H}}=214.3 \mathrm{MeV}$. The horizontal lines are the corresponding upper bounds in Eqs. (7) and (34).

sponding branching ratio of the isospin-related mode $K_{L} \rightarrow \pi^{0} \mathcal{H}$.

For $\Sigma^{+} \rightarrow p \mathcal{H}$, the total amplitude results from adding the contributions in Eqs. (29) and (44). Including only the real part of amplitudes again, and using $r_{8} \simeq-5.1$ determined above, we plot in Fig. 4 the branching ratio in the $\mathrm{SM}$ as a function of $D-F$ for the range in Eq. (30). This figure shows that the curve resulting from the $P$-wave fit using Eqs. (46) and (47) satisfies the HyperCP constraints for certain $D-F$ values.

In Figs. 5 and 6, we display the corresponding branching ratios in the 2HDM II obtained using the parameters in Eqs. (24) and (25). In contrast to the SM case, here $\mathcal{B}\left(K^{+} \rightarrow \pi^{+} \mathcal{H}\right)=0$ when $r_{8} \simeq 6.7$, but the vanishing of the $K_{L}$ rate occurs at a different $r_{8}$ value due to $\mathcal{M}_{4 q}\left(K_{L} \rightarrow \pi^{0} \mathcal{H}\right)$ and $-\operatorname{Re} \mathcal{M}_{4 q}\left(K^{+} \rightarrow \pi^{+} \mathcal{H}\right)$ being unequal with $k_{u} \neq k_{d}$ in Eq. (43). ${ }^{3}$ As a consequence, the two kaon constraints cannot be satisfied simultaneously. Furthermore, the $\Sigma^{+} \rightarrow p \mathcal{H}$ curve that falls within the HyperCP limits is the one resulting from the $S$-wave fit using Eqs. (46) and (47).

To summarize this section, a light Higgs boson in the $\mathrm{SM}$ can be made compatible with the empirical bounds for $\Sigma^{+} \rightarrow p \mathcal{H}$, while satisfying the constraints from $K \rightarrow$ $\pi \mathcal{H}$, if the real part of the two-quark (penguin) contribution to the respective amplitudes is combined with the fourquark contribution. Moreover, in the 2HDM such a particle can satisfy all these constraints if its diagonal couplings to the up- and down-type quarks are the same. For this to happen in either model, it is necessary for the two amplitudes to cancel precisely, and we have shown that this is possible for certain values of the hadronic constants $\tilde{\gamma}_{8}$, $h_{D}-h_{F}$, and $D-F$. Although $\tilde{\gamma}_{8}$ is not known, unlike

\footnotetext{
${ }^{3}$ We note that, although $\tilde{\gamma}_{8}$ is not known from experiment, there are model calculations $[8,26]$ of it yielding $\left|\tilde{\gamma}_{8} / \gamma_{8}\right| \sim 0.2$. This would make the kaon rates greatly exceed their bounds, as can be seen from Figs. 3 and 5.
}

PHYSICAL REVIEW D 74, 115015 (2006)

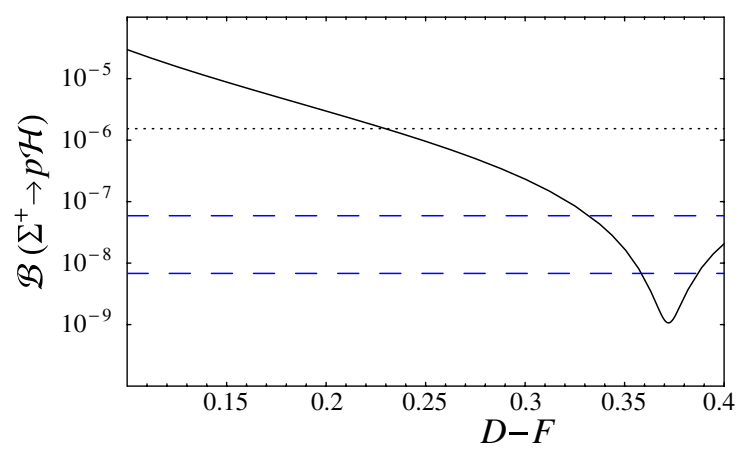

FIG. 4 (color online). Contribution of the real part of the total amplitude for $\Sigma^{+} \rightarrow p \mathcal{H}$ to its branching ratio in the $\mathrm{SM}$ as a function of $D-F$ for $m_{\mathcal{H}}=214.3 \mathrm{MeV}$ and $r_{8} \simeq-5.1$. The solid (dotted) curve corresponds to $h_{D}-h_{F}$ extracted from the $P$-wave ( $S$-wave) fit to the $\Sigma^{+} \rightarrow p \pi^{0}$ data using Eqs. (46) and (47). The dashed lines correspond to the upper and lower bounds in the HyperCP result.

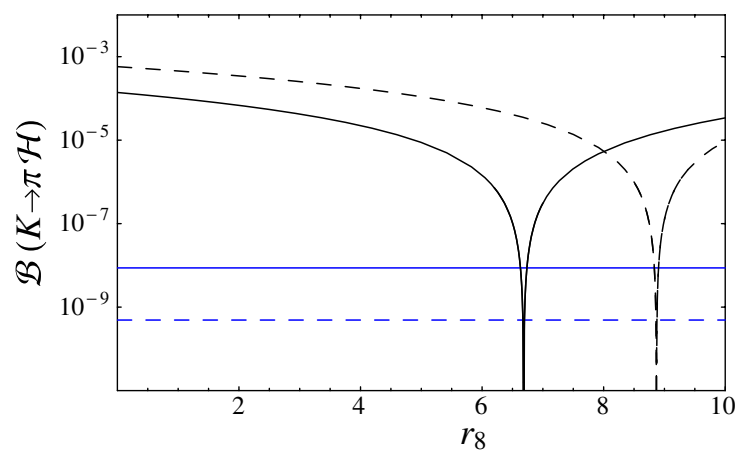

FIG. 5 (color online). Contributions of real parts of total amplitudes for $K^{+} \rightarrow \pi^{+} \mathcal{H}$ (solid curve) and $K_{L} \rightarrow \pi^{0} \mathcal{H}$ (dashed curve) in the 2HDM II to their branching ratios as functions of $r_{8}=\tilde{\gamma}_{8} / \gamma_{8}$ for $m_{\mathcal{H}}=214.3 \mathrm{MeV}$ and the parameters in Eq. (25). The horizontal lines indicate the upper bounds in Eqs. (7) and (34).

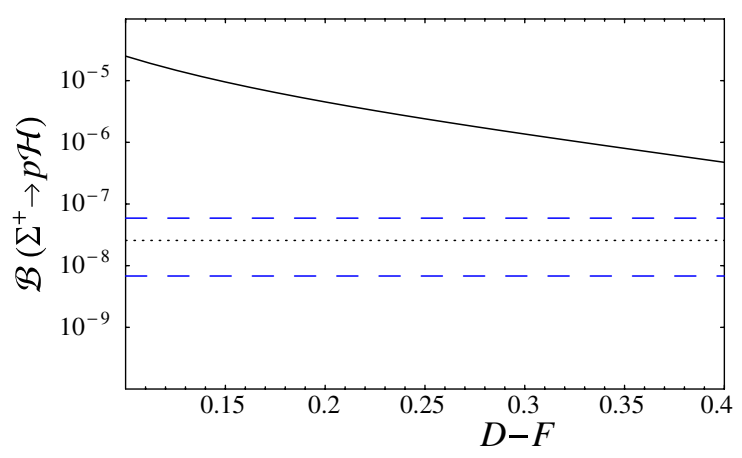

FIG. 6 (color online). Contribution of the real part of the total amplitude for $\Sigma^{+} \rightarrow p \mathcal{H}$ to its branching ratio in the $2 \mathrm{HDM}$ as a function of $D-F$ for $m_{\mathcal{H}}=214.3 \mathrm{MeV}$ and the parameters in Eq. (25). The solid (dotted) curve corresponds to $h_{D}-h_{F}$ extracted from the $P$-wave ( $S$-wave) fit to the $\Sigma^{+} \rightarrow p \pi^{0}$ data using Eqs. (46) and (47). The dashed lines correspond to the upper and lower bounds from the HyperCP result. 
$h_{D}-h_{F}$ and $D-F$ which are extractable from hyperon nonleptonic and semileptonic decays [23], it has a definite value in the SM and cannot be fine-tuned. We note that in all the $\Sigma^{+} \rightarrow p \mathcal{H}$ cases discussed above the $\bar{p} \gamma_{5} \Sigma^{+}$term in the amplitude is small compared to the $\bar{p} \Sigma^{+}$term and that, therefore, the $\tilde{\gamma}_{8}$ contributions are important only in the kaon cases. We also note that flipping the signs of $A_{p \pi^{0}}$ and $B_{p \pi^{0}}$ in Eq. (47), whose overall sign is not fixed by experiment, would prevent the cancellation in the hyperon case from occurring and thus result in rates much above the bounds.

It turns out that the imaginary part of the penguin amplitude is sufficient to eliminate these scalar particles as candidates for the HyperCP events, as it cannot be canceled by the four-quark amplitudes [29], having a size of

$$
\left|\operatorname{Im} g_{\mathcal{H}}\right| \sim 5.8 \times 10^{-7},
$$

much larger than allowed by Eq. (9) with $\operatorname{Im} M_{4 K}=0$. The scaling of the penguin amplitude to the $B$-meson system is also incompatible with the $b \rightarrow s X$ bound. In the SM

$$
g_{\mathcal{H}}^{\prime}=\frac{3 G_{F}}{16 \sqrt{2} \pi^{2}} \sum_{q=u, c, t} m_{q}^{2} V_{q s}^{*} V_{q b} \sim-1.7 \times 10^{-4},
$$

which is much larger than allowed by Eq. (20). In the $2 \mathrm{HDM}$, the relative size is also too large: $\left|g_{\mathcal{H}^{\prime}}^{\prime} / g_{\mathcal{H}}\right| \sim\left|V_{t b} / V_{t d}\right|{ }^{4}$

Both of these problems are associated with a structure in which the Higgs-penguin amplitude is dominated by diagrams with up-type quarks and $W$ bosons in the loops. It may be possible to remedy these problems in models with additional contributions to the penguin, for example, from supersymmetric (SUSY) partners. If the penguin can be sufficiently suppressed, Eqs. (44) and (48) suggest that models in which $k_{W} \sim k_{G}$ could satisfy the kaon bounds while being able to account for the HyperCP result.

\section{PSEUDOSCALAR HIGGS BOSON}

We now consider the possibility that the new particle is a light $C P$-odd pseudoscalar, $\mathcal{A}$, in the two-Higgs-doublet model. Specifically, we do so in types I and II of the model.

\section{A. Two-quark $|\Delta S|=1$ interactions}

The two-quark flavor-changing couplings of $\mathcal{A}$ in the $2 \mathrm{HDM}$ are induced at one loop and have been evaluated in Refs. [31,32]. The effective Lagrangian is the same in

\footnotetext{
${ }^{4}$ One could arrive at a similar conclusion about $\mathcal{H}$ in the 2HDM II by analyzing the decay $\eta \rightarrow \pi^{0} \mathcal{H}$, whose amplitude depends on the four-quark parameters $k_{d}-k_{u}$ [27]. Thus, from the $90 \%$-C.L. bound $\mathcal{B}\left(\eta \rightarrow \pi^{0} \mathcal{H}\right)<5 \times 10^{-6}$ [30], one extracts $\left|k_{d}-k_{u}\right|<0.45$ for $m_{\mathcal{H}}=214.3 \mathrm{MeV}$, which is incompatible with the limit derived from Eq. (43) plus the LEP constraint $\sin ^{2}(\beta-\alpha)<0.06 \quad[21]$, namely $\left|k_{d}-k_{u}\right|=$ $|2 \cos (\alpha-\beta) / \sin (2 \beta)|>1.9$.
}

types I and II of the model and can be written as $\mathcal{L}_{\mathcal{A} s d}$ in Eq. (1b), where

$$
g_{\mathcal{A}}=\frac{G_{F}}{16 \sqrt{2} \pi^{2}} \sum_{q=u, c, t} m_{q}^{2} V_{q d}^{*} V_{q s}\left(\frac{A_{1}(q)}{\tan \beta}+\frac{A_{2}(q)}{\tan ^{3} \beta}\right),
$$

with $A_{1,2}(q)$ being functions of $m_{q}, m_{W}$, and $m_{H^{+}}$, whose expressions can be found in Ref. [31]. The leading-order chiral realization of $\mathcal{L}_{\mathcal{A} s d}$ is then

$$
\begin{aligned}
\mathcal{L}_{\mathcal{A}}= & b_{D}\left\langle\bar{B}\left\{h_{\mathcal{A}}, B\right\}\right\rangle+b_{F}\left\langle\bar{B}\left[h_{\mathcal{A}}, B\right]\right\rangle+b_{0}\left\langle h_{\mathcal{A}}\right\rangle\langle\bar{B} B\rangle \\
& +\frac{1}{2} f^{2} B_{0}\left\langle h_{\mathcal{A}}\right\rangle+\text { H.c. }
\end{aligned}
$$

where

$$
h_{\mathcal{A}}=-2 i g_{\mathcal{A}}\left(\xi^{\dagger} h M \xi^{\dagger}-\xi M h \xi\right) \frac{\mathcal{A}}{v} .
$$

The leading-order diagrams for $K \rightarrow \pi \mathcal{A}$ and $\Sigma^{+} \rightarrow$ $p \mathcal{A}$ arising from $\mathcal{L}_{\mathcal{A}}$, plus $\mathcal{L}_{\mathrm{s}}$, are similar to those in the case of standard-model Higgs bosons, displayed in Fig. 1. The resulting amplitudes are

$$
\begin{aligned}
\mathcal{M}_{2 q}\left(K^{+} \rightarrow \pi^{+} \mathcal{A}\right) & =-\sqrt{2} \mathcal{M}_{2 q}\left(K^{0} \rightarrow \pi^{0} \mathcal{A}\right) \\
& =i g_{\mathcal{A}} \frac{m_{K}^{2}-m_{\pi}^{2}}{v}
\end{aligned}
$$

$$
\begin{aligned}
\mathcal{M}_{2 q}\left(\Sigma^{+} \rightarrow p \mathcal{A}\right)= & i g_{\mathcal{A}} \frac{m_{\Sigma}-m_{N}}{v} \bar{p} \Sigma^{+}-i g_{\mathcal{A}}(D-F) \\
& \times \frac{m_{\Sigma}+m_{N}}{v} \frac{m_{K}^{2}}{m_{K}^{2}-m_{\mathcal{A}}^{2}} \bar{p} \gamma_{5} \Sigma^{+} .
\end{aligned}
$$

\section{B. Four-quark $|\Delta S|=1$ interactions}

The diagonal couplings of $\mathcal{A}$ to light quarks in the 2HDM are described by [32]

$$
\mathcal{L}_{\mathcal{A} q q}=-\bar{q} \tilde{M} \gamma_{5} q \frac{i \mathcal{A}}{v}=-\bar{q}_{\mathrm{L}} \tilde{M} q_{\mathrm{R}} \frac{i \mathcal{A}}{v}+\text { H.c., }
$$

where

$$
q=\left(\begin{array}{lll}
u & d & s
\end{array}\right)^{\mathrm{T}}, \quad \tilde{M}=\operatorname{diag}\left(l_{u} \hat{m}, l_{d} \hat{m}, l_{d} m_{s}\right),
$$

with

$$
\begin{gathered}
l_{u}=-l_{d}=-\cot \beta \quad \text { in the 2HDM I, } \\
l_{u}=-\cot \beta, \quad l_{d}=-\tan \beta \quad \text { in the 2HDM II. }
\end{gathered}
$$

Since the Lagrangian for the quark masses is $\mathcal{L}_{q}=$ $-\bar{q}_{\mathrm{L}} M q_{\mathrm{R}}+$ H.c., the effect of $\mathcal{L}_{q q \mathcal{A}}$ on interactions described by $\mathcal{L}_{\mathrm{s}, \mathrm{w}}$ can be taken into account using $\mathcal{L}_{\mathrm{s}, \mathrm{w}}$ and substituting $M$ with $\tilde{M} i \mathcal{A} / v$ [10]. The resulting Lagrangians are 


$$
\begin{aligned}
\mathcal{L}_{\mathrm{s}}^{\mathcal{A}}= & \left(b_{D}\left\langle\bar{B}\left\{\tilde{M}_{-}, B\right\}\right\rangle+b_{F}\left\langle\bar{B}\left[\tilde{M}_{-}, B\right]\right\rangle+b_{0}\left\langle\tilde{M}_{-}\right\rangle\langle\bar{B} B\rangle\right. \\
+ & \left.\frac{1}{2} f^{2} B_{0}\left\langle\tilde{M}_{-}\right\rangle\right) \frac{i \mathcal{A}}{v}, \\
& \mathcal{L}_{\mathrm{w}}^{\mathcal{A}}=2 \tilde{\gamma}_{8} f^{2} B_{0}\left\langle h \xi \tilde{M}_{-} \xi^{\dagger}\right\rangle \frac{i \mathcal{A}}{v}+\text { H.c. }
\end{aligned}
$$

where

$$
\tilde{M}_{-}=\xi^{\dagger} \tilde{M} \xi^{\dagger}-\xi \tilde{M}^{\dagger} \xi
$$

In addition, if the $\mathrm{SU}(3)$ singlet $\eta_{1}$ is included in $\mathcal{L}_{\mathrm{s}, \mathrm{w}}^{(\mathcal{A})}$ by replacing $\Sigma$ with $\Sigma \exp \left(i \sqrt{2 / 3} \eta_{1} / f\right)$, the coupling of $\mathcal{A}$ to two gluons via the axial anomaly gives rise to [10]

$$
\begin{aligned}
\mathcal{L}_{\eta_{1} \mathcal{A}}= & -\frac{1}{2}\left(m_{\eta_{1}}^{2}-\frac{2}{3} m_{K}^{2}-\frac{1}{3} m_{\pi}^{2}\right) \\
& \times\left[\eta_{1}+\frac{f \mathcal{A}}{\sqrt{6} v}\left(2 l_{u}+l_{d}\right)\right]^{2},
\end{aligned}
$$

which modifies the $\eta_{1}-\mathcal{A}$ mixing generated by $\mathcal{L}_{\mathrm{s}}^{\mathcal{A}}$.

From $\mathcal{L}_{\mathrm{s}, \mathrm{w}}^{(\mathcal{A})}$, we derive the leading-order diagrams shown in Fig. 7 for $K \rightarrow \pi \mathcal{A}$, where

$$
\eta=\eta_{8} \cos \theta-\eta_{1} \sin \theta, \quad \eta^{\prime}=\eta_{8} \sin \theta+\eta_{1} \cos \theta .
$$

The resulting amplitudes are

$$
\begin{aligned}
\mathcal{M}_{4 q}\left(K^{+} \rightarrow \pi^{+} \mathcal{A}\right)= & \frac{i \gamma_{8}\left(l_{u}-l_{d}\right) m_{\pi}^{2}}{2 v}+i \gamma_{8}\left[\left(2 m_{K}^{2}+m_{\pi}^{2}-3 m_{\mathcal{A}}^{2}\right) c_{\theta}-\sqrt{8}\left(m_{K}^{2}-m_{\pi}^{2}\right) s_{\theta}\right] \\
& \times \frac{\left[4 l_{d} m_{K}^{2}-\left(3 l_{d}+l_{u}\right) m_{\pi}^{2}\right] c_{\theta}+\sqrt{2}\left[2 l_{d} m_{K}^{2}+l_{u} m_{\pi}^{2}-\left(l_{d}+2 l_{u}\right) \tilde{m}_{0}^{2}\right] s_{\theta}}{6\left(m_{\eta}^{2}-m_{\mathcal{A}}^{2}\right) v}+i \gamma_{8}\left[\left(2 m_{K}^{2}+m_{\pi}^{2}-3 m_{\mathcal{A}}^{2}\right) s_{\theta}\right. \\
& \left.+\sqrt{8}\left(m_{K}^{2}-m_{\pi}^{2}\right) c_{\theta}\right] \frac{\left[4 l_{d} m_{K}^{2}-\left(3 l_{d}+l_{u}\right) m_{\pi}^{2}\right] s_{\theta}-\sqrt{2}\left[2 l_{d} m_{K}^{2}+l_{u} m_{\pi}^{2}-\left(l_{d}+2 l_{u}\right) \tilde{m}_{0}^{2}\right] c_{\theta}}{6\left(m_{\eta^{\prime}}^{2}-m_{\mathcal{A}}^{2}\right) v} \\
\mathcal{M}_{4 q}\left(K^{0} \rightarrow \pi^{0} \mathcal{A}\right)= & \frac{i \gamma_{8}\left(l_{u}-l_{d}\right)\left(2 m_{K}^{2}-m_{\pi}^{2}-m_{\mathcal{A}}^{2}\right) m_{\pi}^{2}}{\sqrt{8}\left(m_{\mathcal{A}}^{2}-m_{\pi}^{2}\right) v}+i \gamma_{8}\left[\left(2 m_{K}^{2}+m_{\pi}^{2}-3 m_{\mathcal{A}}^{2}\right) c_{\theta}-\sqrt{8}\left(m_{K}^{2}-m_{\pi}^{2}\right) s_{\theta}\right] \\
& \times \frac{\left[4 l_{d} m_{K}^{2}-\left(3 l_{d}+l_{u}\right) m_{\pi}^{2}\right] c_{\theta}+\sqrt{2}\left[2 l_{d} m_{K}^{2}+l_{u} m_{\pi}^{2}-\left(l_{d}+2 l_{u}\right) \tilde{m}_{0}^{2}\right] s_{\theta}}{6 \sqrt{2}\left(m_{\mathcal{A}}^{2}-m_{\eta}^{2}\right) v} \\
& +i \gamma_{8}\left[\left(2 m_{K}^{2}+m_{\pi}^{2}-3 m_{\mathcal{A}}^{2}\right) s_{\theta}+\sqrt{8}\left(m_{K}^{2}-m_{\pi}^{2}\right) c_{\theta}\right] \\
& \times \frac{\left[4 l_{d} m_{K}^{2}-\left(3 l_{d}+l_{u}\right) m_{\pi}^{2}\right] s_{\theta}-\sqrt{2}\left[2 l_{d} m_{K}^{2}+l_{u} m_{\pi}^{2}-\left(l_{d}+2 l_{u}\right) \tilde{m}_{0}^{2}\right] c_{\theta}}{6 \sqrt{2}\left(m_{\mathcal{A}}^{2}-m_{\eta^{\prime}}^{2}\right) v} \\
&
\end{aligned}
$$

where

$$
c_{\theta}=\cos \theta, \quad s_{\theta}=\sin \theta, \quad \tilde{m}_{0}^{2}=m_{\eta_{1}}^{2}-\frac{2}{3} m_{K}^{2}-\frac{1}{3} m_{\pi}^{2} .
$$

The $\tilde{\gamma}_{8}$ contributions to this amplitude cancel completely, as already noted in Ref. [10]. Numerically, $\tilde{m}_{0}^{2} \simeq 819 \mathrm{MeV}$ from fitting to the $\eta^{\prime}$ mass after diagonalizing the $\eta_{8,1}$ masses derived from the Lagrangians in Eqs. (28) and (64), and consequently $\theta \simeq-19.7^{\circ}$.

The leading-order four-quark contributions to $\Sigma^{+} \rightarrow p \mathcal{A}$ arise from the diagrams in Fig. 8 and can be expressed as
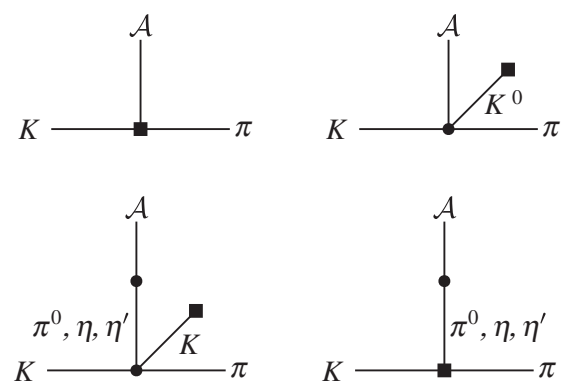

FIG. 7. Diagrams contributing to $K \rightarrow \pi \mathcal{A}$ arising from the four-quark operators. The dots come from $\mathcal{L}_{\mathrm{s}}^{(\mathcal{A})}$ in Eqs. (28) and (61), whereas the square vertices are from $\mathcal{L}_{\mathrm{w}}^{(\mathcal{A})}$ in Eqs. (36) and (62).
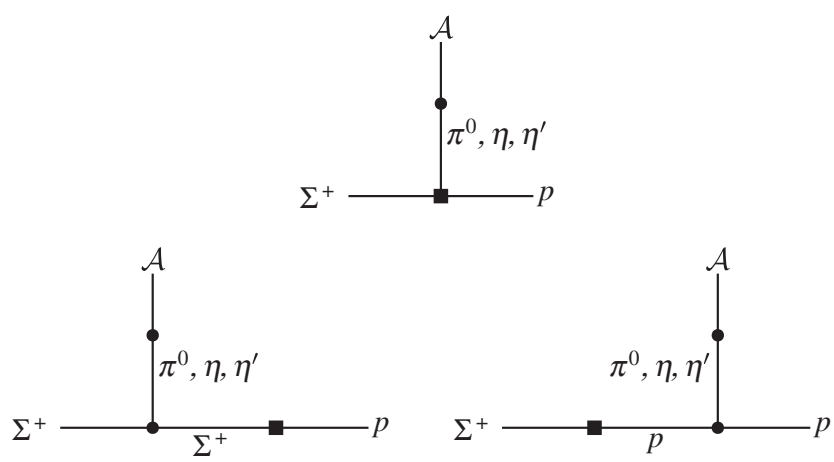

FIG. 8. Diagrams contributing to $\Sigma^{+} \rightarrow p \mathcal{A}$ arising from the four-quark operators. The square vertices come from $\mathcal{L}_{\mathrm{w}}$ in Eq. (36), whereas the dots are from the Lagrangians in Eqs. (28), (61), and (64). 
where

$$
\mathcal{M}_{4 q}\left(\Sigma^{+} \rightarrow p \mathcal{A}\right)=i \bar{p}\left(A_{p \mathcal{A}}-B_{p \mathcal{A}} \gamma_{5}\right) \Sigma^{+},
$$

$$
\begin{aligned}
A_{p \mathcal{A}}= & \frac{f A_{p \pi^{0}}\left(l_{d}-l_{u}\right) m_{\pi}^{2}}{2\left(m_{\mathcal{A}}^{2}-m_{\pi}^{2}\right) v}+\frac{f A_{p \pi^{0}}\left\{\left[4 l_{d} m_{K}^{2}-\left(3 l_{d}+l_{u}\right) m_{\pi}^{2}\right] c_{\theta}^{2}+\sqrt{2}\left[2 l_{d} m_{K}^{2}+l_{u} m_{\pi}^{2}-\left(l_{d}+2 l_{u}\right) \tilde{m}_{0}^{2}\right] c_{\theta} s_{\theta}\right\}}{2\left(m_{\eta}^{2}-m_{\mathcal{A}}^{2}\right) v} \\
& +\frac{f A_{p \pi^{0}}\left\{\left[4 l_{d} m_{K}^{2}-\left(3 l_{d}+l_{u}\right) m_{\pi}^{2}\right] s_{\theta}^{2}-\sqrt{2}\left[2 l_{d} m_{K}^{2}+l_{u} m_{\pi}^{2}-\left(l_{d}+2 l_{u}\right) \tilde{m}_{0}^{2}\right] c_{\theta} s_{\theta}\right\}}{2\left(m_{\eta^{\prime}}^{2}-m_{\mathcal{A}}^{2}\right) v}, \\
B_{p \mathcal{A}}= & \frac{f B_{p \pi^{0}}\left(l_{d}-l_{u}\right) m_{\pi}^{2}}{2\left(m_{\mathcal{A}}^{2}-m_{\pi}^{2}\right) v}+\frac{f B_{p \pi^{0}}\left\{\left[4 l_{d} m_{K}^{2}-\left(3 l_{d}+l_{u}\right) m_{\pi}^{2}\right] c_{\theta}^{2}+\sqrt{2}\left[2 l_{d} m_{K}^{2}+l_{u} m_{\pi}^{2}-\left(l_{d}+2 l_{u}\right) \tilde{m}_{0}^{2}\right] c_{\theta} s_{\theta}\right\}}{2\left(m_{\eta}^{2}-m_{\mathcal{A}}^{2}\right) v} \\
& +\frac{f B_{p \pi^{0}}\left\{\left[4 l_{d} m_{K}^{2}-\left(l_{d}+l_{u}\right) m_{\pi}^{2}\right] s_{\theta}^{2}-\sqrt{2}\left[2 l_{d} m_{K}^{2}+l_{u} m_{\pi}^{2}-\left(l_{d}+2 l_{u}\right) \tilde{m}_{0}^{2}\right] c_{\theta} s_{\theta}\right\}}{2\left(m_{\eta^{\prime}}^{2}-m_{\mathcal{A}}^{2}\right) v},
\end{aligned}
$$

where $A_{p \pi^{0}}$ and $B_{p \pi^{0}}$ are given in Eq. (46). We note that contributions with $\gamma_{8}$ or $\tilde{\gamma}_{8}$ appear only at next-to-leading order.

\section{Total contributions}

The total amplitudes for $K \rightarrow \pi \mathcal{A}$ result from adding the contributions in Eqs. (55) and (66). If the $C P$-violating terms in the amplitudes are ignored, it is possible for the two-quark and four-quark contributions to cancel. We show this possibility in Fig. 9, where we plot the resulting branching ratios as functions of the charged-Higgs-boson mass for $m_{\mathcal{A}}=214.3 \mathrm{MeV}$ and different $\tan \beta$ values in the 2 versions of the $2 \mathrm{HDM}$. The total amplitude for $\Sigma^{+} \rightarrow$ $p \mathcal{A}$ is the sum of the contributions in Eqs. (56) and (68). If the experimental values of $A_{p \pi^{0}}$ and $B_{p \pi^{0}}$ in Eq. (47), as well as the middle value $D-F=0.25$, are used in the total amplitude, the resulting branching ratios in the 2HDM are displayed in Fig. 10.

We have found that only one of the kaon bounds can be satisfied if the HyperCP result is assumed to be mediated by $\mathcal{A}$ in the $2 \mathrm{HDM}$. However, for certain $\tan \beta$ and $m_{H^{+}}$ values near the ones indicated in Figs. 9 and 10, all the kaon and hyperon constraints can be nearly satisfied simultaneously. Part of the difficulty in satisfying all of the constraints lies with the vanishing of the $K^{+}$and $K_{S}$ rates occurring at different $m_{H^{+}}$values, which is due to

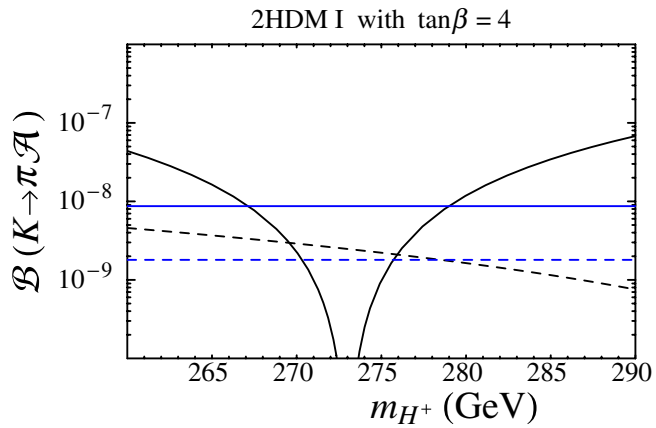

$\mathcal{M}_{4 q}\left(K_{S} \rightarrow \pi^{0} \mathcal{H}\right)$ and $-\operatorname{Re} \mathcal{M}_{4 q}\left(K^{+} \rightarrow \pi^{+} \mathcal{H}\right)$ being unequal with $l_{u} \neq l_{d}$ in Eqs. (59) and (60). We note that the situation is not much different if the signs of $A_{p \pi^{0}}$ and $B_{p \pi^{0}}$ in Eq. (47) are both flipped.

To summarize this section, we have found that it is possible for the real part of the penguin amplitude to cancel against the four-quark amplitude to approximately satisfy the kaon bounds while explaining the HyperCP observation with a $2 \mathrm{HDM}$ pseudoscalar. Unlike the scalar case, there is no free hadronic parameter at leading order in $\chi \mathrm{PT}$ in this case. The cancellation must happen as a function of the short-distance parameters that determine the size of the amplitudes.

A feature shared by scalars and pseudoscalars in the $2 \mathrm{HDM}$ is that the imaginary part of the penguin amplitude is incompatible with the kaon bounds in Eq. (9) and has no counterpart that could cancel it in the four-quark amplitude. A related problem is that the scaling of the penguin amplitude to the $B$ system is also incompatible with observation.

In view of these flaws, it is tempting to search for a model in which the penguin amplitudes are completely suppressed, and the 2HDM II seems to allow us to do that. In the 2HDM II the penguin amplitudes are proportional to $l_{u}$, whereas the four-quark amplitudes receive contributions from both $l_{u}$ and $l_{d}$ in Eq. (60). Thus the

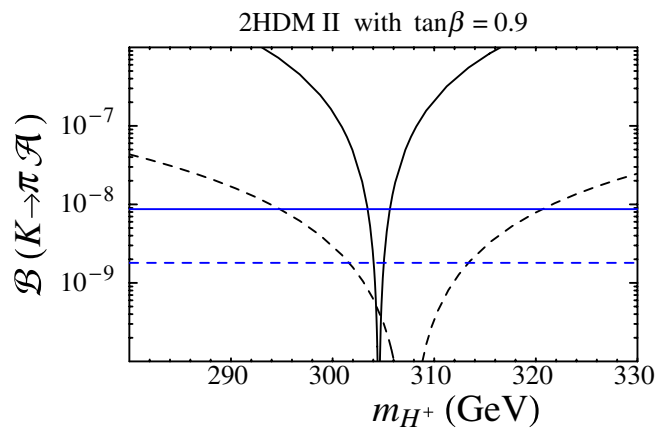

FIG. 9 (color online). Contributions of real parts of total amplitudes for $K^{+} \rightarrow \pi^{+} \mathcal{A}$ (solid curve) and $K_{S} \rightarrow \pi^{0} \mathcal{A}$ (dashed curve) in the 2HDM to their branching ratios as functions of charged-Higgs-boson mass for $m_{\mathcal{A}}=214.3 \mathrm{MeV}$ and $\tan \beta=4(0.9)$ in type I(II) of the model. The horizontal lines indicate the upper bounds in Eqs. (7) and (8). 


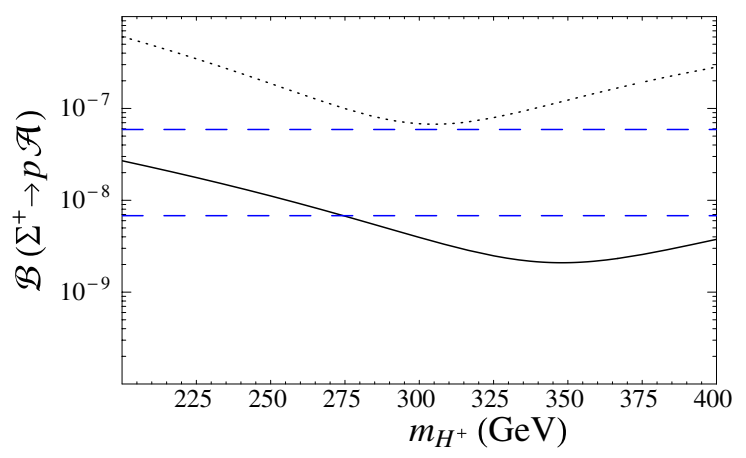

FIG. 10 (color online). Contribution of the real part of the total amplitude for $\Sigma^{+} \rightarrow p \mathcal{A}$ to its branching ratio in the $2 \mathrm{HDM} \mathrm{I}$ (solid curve) and II (dotted curve) as a function of chargedHiggs-boson mass for $m_{\mathcal{A}}=214.3 \mathrm{MeV}$ and $\tan \beta=4(0.9)$ in type I(II). The dashed lines indicate the bounds from the HyperCP result.

model in the large- $\tan \beta$ limit has $l_{u} \rightarrow 0$. Unfortunately, in this limit $l_{d}$ induces four-quark amplitudes resulting in

$$
\frac{\mathcal{B}\left(\Sigma^{+} \rightarrow p \mathcal{A}\right)}{\mathcal{B}\left(K^{+} \rightarrow \pi^{+} X\right)} \rightarrow 0.025,
$$

which is inconsistent with Eqs. (9) and (13). In the 2HDM I, which has $l_{u}$ and $l_{d}$ given in Eq. (59), the four-quark amplitudes alone yield

$$
\frac{\mathcal{B}_{4 q}\left(\Sigma^{+} \rightarrow p \mathcal{A}\right)}{\mathcal{B}_{4 q}\left(K^{+} \rightarrow \pi^{+} X\right)}=0.53
$$

for all values of $\tan \beta$, which is consistent with Eqs. (9) and (13). However, in this case it is the penguin amplitude that eliminates the pseudoscalar as a possible HyperCP candidate.

These results suggest the ingredients of a model that can satisfy all constraints. It is necessary for the penguin amplitudes to be dominated by additional particles, such as SUSY partners, in such a way that $g_{\mathcal{A}}$ is not proportional to top-quark CKM angles. We have sketched a scenario where this happens in Ref. [33].

\section{SUMMARY AND CONCLUSIONS}

We have summarized the existing constraints on the production of a light Higgs boson in kaon and $B$-meson decays, as well as the implication of attributing the HyperCP events to the production of a light Higgs boson in hyperon decay.

Production rates for such a particle in kaon and hyperon decays receive contributions from two- and four-quark operators that can be comparable in some cases. We have investigated the interplay of both production mechanisms with the aid of leading-order chiral perturbation theory. To this effect, we have implemented the low-energy theorems governing the couplings of light (pseudo)scalars to hadrons at leading order in baryon $\chi \mathrm{PT}$, generalizing existing studies for kaon decay.
We first discussed the case of a scalar Higgs boson. We found that the leading-order amplitudes in both kaon and hyperon decays depend on an unknown low-energy constant $\tilde{\gamma}_{8}$, as well as known constants from the hyperon sector. This constant is connected to a weak-mass term in the chiral Lagrangian that can be rotated away for processes that involve only pseudo-Goldstone bosons and is, therefore, unknown. We applied our results to the process $\Sigma^{+} \rightarrow p X$ relevant to the HyperCP observation of $\Sigma^{+} \rightarrow$ $p \mu^{+} \mu^{-}$. We showed that the two-quark contributions in the SM and its 2HDM extensions are too large to explain the HyperCP observation. However, we also showed that there can be cancellations between the $C P$-conserving twoand four-quark contributions to this process that lead to a rate comparable in size to the HyperCP observation for both the SM and the 2HDM. Such cancellations occur for a certain range of known constants from the hyperon sector, the effect of $\tilde{\gamma}_{8}$ being small. In both cases, however, the two-quark penguin contribution has an imaginary $(\mathrm{CP}$-violating $)$ part that is too large to be compatible with the HyperCP result. In the SM and in the 2HDM, the four-quark contributions have a $C P$-violating part that is much smaller than that of the penguin amplitude and hence these models are ruled out as explanations for the HyperCP observation. More general models with additional $C P$-violating phases may be able to address this issue. In addition, in these models the scaling of the twoquark operator to the $B$ system is incompatible with the nonobservation of a light scalar in $B$ decay.

We then discussed the case of a pseudoscalar Higgs boson in the 2HDM. In this case we computed the leading-order amplitudes in $\chi \mathrm{PT}$ and included, as well, certain higher-order terms mediated by the $\eta^{\prime}$ state. The resulting amplitudes for both kaon and hyperon decays do not depend on any unknown hadronic parameters. In particular, they do not depend on $\tilde{\gamma}_{8}$, as observed in Ref. [10]. We then applied our results to the $\Sigma^{+} \rightarrow p \mathcal{A}$ process. Once again we found that the real part of the amplitude can be consistent with the HyperCP observation for a certain range of parameters in the $2 \mathrm{HDM}(\tan \beta$ and $m_{H^{+}}$), but that the imaginary part of the penguin amplitude is too large. The scaling of the two-quark operator to the $B$ system also produces a $B \rightarrow X_{s} \mathcal{A}$ rate that is too large. Both of these problems can be solved in more general models that modify the phase and scaling with CKM angles of the two-quark operator.

In conclusion, we have shown that it is possible to interpret the HyperCP observation as evidence for a light Higgs boson, although it is not easy to arrange this in a model. Typical Higgs-penguin operators have three problems:

(a) if they have the right size to fit the HyperCP observation, they induce $K \rightarrow \pi X$ at rates larger than the existing bounds;

(b) if they are dominated by loop diagrams involving up-type quarks and $W$ bosons, they have a $C P$ phase 
that is too large;

(c) if they are dominated by loop diagrams involving up-type quarks and $W$ bosons, their scaling to the $B$ system is incompatible with the nonobservation of $B \rightarrow X_{s} X$.

We have found in this paper that (a) can be solved in some cases by the addition of the effects of four-quark operators. We have suggested that more general models may be constructed to solve (b) and (c). To show that this is possible, we have constructed a specific example in Ref. [33].

Disregarding existing bounds from kaon and $B$-meson decays, we have shown that many light Higgs bosons have couplings of the right size to explain the HyperCP observation. We think this is sufficiently intriguing to warrant a revisiting of the kaon and $B$-decay results. In particular, the $B$ factories are still operational and could reanalyze the very low $m_{\mu \mu}$ invariant-mass region in their measurements of $B \rightarrow X_{s} \mu^{+} \mu^{-}$modes. The NA48 experiment might also be able to revisit the kaon modes.

\section{ACKNOWLEDGMENTS}

The work of X. G. H. was supported in part by NSC and NCTS. The work of G. V. was supported in part by DOE under Contract No. DE-FG02-01ER41155. We thank Laurence Littenberg and Rainer Wanke for useful discussions on the kaon bounds and Soeren Prell for useful discussions on the $B$ bounds.
[1] H. Park et al. (HyperCP Collaboration), Phys. Rev. Lett. 94, 021801 (2005).

[2] X. G. He, J. Tandean, and G. Valencia, Phys. Rev. D 72, 074003 (2005).

[3] X. G. He, J. Tandean, and G. Valencia, Phys. Lett. B 631, 100 (2005).

[4] N. G. Deshpande, G. Eilam, and J. Jiang, Phys. Lett. B 632, 212 (2006).

[5] C. Q. Geng and Y. K. Hsiao, Phys. Lett. B 632, 215 (2006).

[6] D. S. Gorbunov and V.A. Rubakov, Phys. Rev. D 64, 054008 (2001); 73, 035002 (2006); S. V. Demidov and D. S. Gorbunov, hep-ph/0610066.

[7] R. S. Chivukula and A. V. Manohar, Phys. Lett. B 207, 86 (1988); 217, 568(E) (1989).

[8] H. Leutwyler and M. A. Shifman, Nucl. Phys. B343, 369 (1990).

[9] See, for example, J.F. Gunion, H.E. Haber, G. L. Kane, and S. Dawson, Report No. SCIPP-89/13, and references therein.

[10] B. Grzadkowski and J. Pawelczyk, Phys. Lett. B 300, 387 (1993).

[11] H. Ma et al. (E865 Collaboration), Phys. Rev. Lett. 84, 2580 (2000).

[12] H. K. Park et al. (HyperCP Collaboration), Phys. Rev. Lett. 88, 111801 (2002).

[13] J. R. Batley et al. (NA48/1 Collaboration), Phys. Lett. B 599, 197 (2004).

[14] E. Barberio et al. (Heavy Flavor Averaging Group), hepex/0603003.

[15] B. Aubert et al. (BABAR Collaboration), Phys. Rev. Lett. 93, 081802 (2004).

[16] M. Iwasaki et al. (Belle Collaboration), Phys. Rev. D 72, 092005 (2005).

[17] R. S. Willey and H. L. Yu, Phys. Rev. D 26, 3086 (1982); B. Grzadkowski and P. Krawczyk, Z. Phys. C 18, 43 (1983); A. Dedes, Mod. Phys. Lett. A 18, 2627 (2003), and references therein.

[18] R. M. Barnett, G. Senjanovic, and D. Wyler, Phys. Rev. D 30, 1529 (1984); C. Q. Geng and J. N. Ng, ibid. 39, 3330 (1989); M.E. Lautenbacher, Nucl. Phys. B347, 120 (1990).

[19] S. Dawson, Nucl. Phys. B339, 19 (1990).

[20] J. Charles et al. (CKMfitter Group), Eur. Phys. J. C 41, 1 (2005). Updated results used in this paper are from http:// ckmfitter.in2p3.fr/, "Results as of FPCP 2006, Vancouver, Canada."

[21] G. Abbiendi et al. (OPAL Collaboration), Eur. Phys. J. C 40, 317 (2005).

[22] J. Gasser and H. Leutwyler, Ann. Phys. (N.Y.) 158, 142 (1984).

[23] J. Bijnens, H. Sonoda, and M. B. Wise, Nucl. Phys. B261, 185 (1985); E. Jenkins and A. V. Manohar, in Effective Field Theories of the Standard Model, edited by U.-G. Meissner (World Scientific, Singapore, 1992).

[24] A. Alavi-Harati et al. (KTEV Collaboration), Phys. Rev. Lett. 84, 5279 (2000).

[25] See, for example, J. F. Donoghue, E. Golowich, and B. R. Holstein, Dynamics of the Standard Model (Cambridge University Press, Cambridge, 1992).

[26] J. Bijnens and J. Prades, J. High Energy Phys. 01 (1999) 023.

[27] J. Prades and A. Pich, Phys. Lett. B 245, 117 (1990); A. Pich, J. Prades, and P. Yepes, Nucl. Phys. B388, 31 (1992).

[28] W.-M. Yao et al. (Particle Data Group), J. Phys. G 33, 1 (2006).

[29] H. Y. Cheng and H. L. Yu, Phys. Rev. D 40, 2980 (1989).

[30] R. I. Dzhelyadin et al., Phys. Lett. 105B, 239 (1981).

[31] J. M. Frere, J. A. M. Vermaseren, and M. B. Gavela, Phys. Lett. 103B, 129 (1981).

[32] L. J. Hall and M. B. Wise, Nucl. Phys. B187, 397 (1981).

[33] X. G. He, J. Tandean, and G. Valencia, hep-ph/0610362. 